\title{
Development of an Orbital Trajectory Analysis Tool
}

\section{Sittiporn Channumsin ${ }^{1, a,}$, Prakasit Udomthanatheera ${ }^{1, \mathrm{~b}}$, Chotika Kositratpatcharasuk $^{1, \mathrm{c}}$, and Manop Aorpimai ${ }^{2, \mathrm{~d}}$}

1 Geo-Informatics and Space Technology Development Agency (Public Organization), GISTDA, 88 M. 9 Thung Sukala Road, Siracha, Chonburi 20230, Thailand

2 Thaipat Satellite Research Center, Mahanakorn University of Technology, 140 Chuam Sampan Road, Nong Chok, Bangkok 105301, Thailand

E-mail: asittiporn@gistda.or.th (Corresponding author), bprakasit.u@gistda.or.th, cchotika@gistda.or.th. dmanop@mut.ac.th

\begin{abstract}
Since Thailand successfully launched the first earth observation satellite (Thaichote) in 2008, the Geo-Informatics and Space Technology Development Agency (GISTDA) has started developing an orbit analysis tool called "EMERALD" to be used for the current and future mission planned by GISTDA. In this paper, we present the development of a satellite orbit control maneuver, which is one of the analysis tools, by providing essential parameters for an orbital trajectory analysis and design. The algorithms are developed and programmed in a convenient graphical user interface (GUI). The results can guarantee a mission and design a desired orbital mission by calculating suitable maneuver parameters to correct the ground track (GT) and local solar time (LST) under control window including the transfer orbit for the good quality of the mission data. The validation results are in good agreement with Quartz++, which is a flight dynamics software developed by EADS ASTRIUM.
\end{abstract}

Keywords: Thaichote satellite, orbit control, flight dynamics program, EMERALD, graphic user interface, orbit analysis tool, mission analysis, station keeping. 


\section{Introduction}

Thaichote is the Thailand's first operational Earth Observation Satellites (THEOS) [1]. It is categorized into a Low Earth Orbit (LEO) satellite at altitude $822 \mathrm{~km}$. The orbit type is a polar, sun-synchronous orbit and station keeping (SK) and there are two major payloads: panchromatic (PAN) and multi-spectral (MS) cameras, which are monochrome and color camera respectively. MS camera consists of red, green, blue and near-infrared (NIR) color model. In order to control SK, ground track (GT) error and local solar time (LST) error are concerned parameters that are necessary to monitor daily by flight dynamics team. Quartz++, the flight dynamics program developed by EADS ASTRIUM, is originally implemented for the operation with the sun workstation machine and run on a UNIX OS system. This program has been employed since the satellite was launched into space in 2008. Based on the accumulated experience and planning, Geo-Informatics and Space Technology Development Agency (GISTDA) has started EMERALD project [2] to develop a tool for mission analysis and design. This project is the cooperation between GISTDA and Mahanakorn University of Technology (MUT) with the main purpose to implement EMERALD instead of Quartz++ and supports the near future satellites in Thailand. The $1^{\text {st }}$ phase has been validated and succeeded to model the space environment and can predict the orbital dynamics of a satellite by using numerical integrator technique. For the $2^{\text {nd }}$ phase, these algorithms are developed in convenient graphic user interface (GUI) as shown in Fig. 1 and provides 6 main modules (orbit determination, event prediction, collision risk assessment, orbit control maneuver (OCM), de-orbit simulation and operation report).

In this paper, we focus on the evaluation of the OCM module that has 5 sub-modules on GUI as shown in Fig. 1. The validation is performed by comparing with Quartz++ and the results are in good agreement.

The rest of this paper is organized as following. The fundamental algorithms of orbit control maneuver $(\mathrm{OCM})$ are introduced in section 2. Section 3 presents the results and GUI of the OCM module. Finally, some conclusions and future works are drawn in section 4.

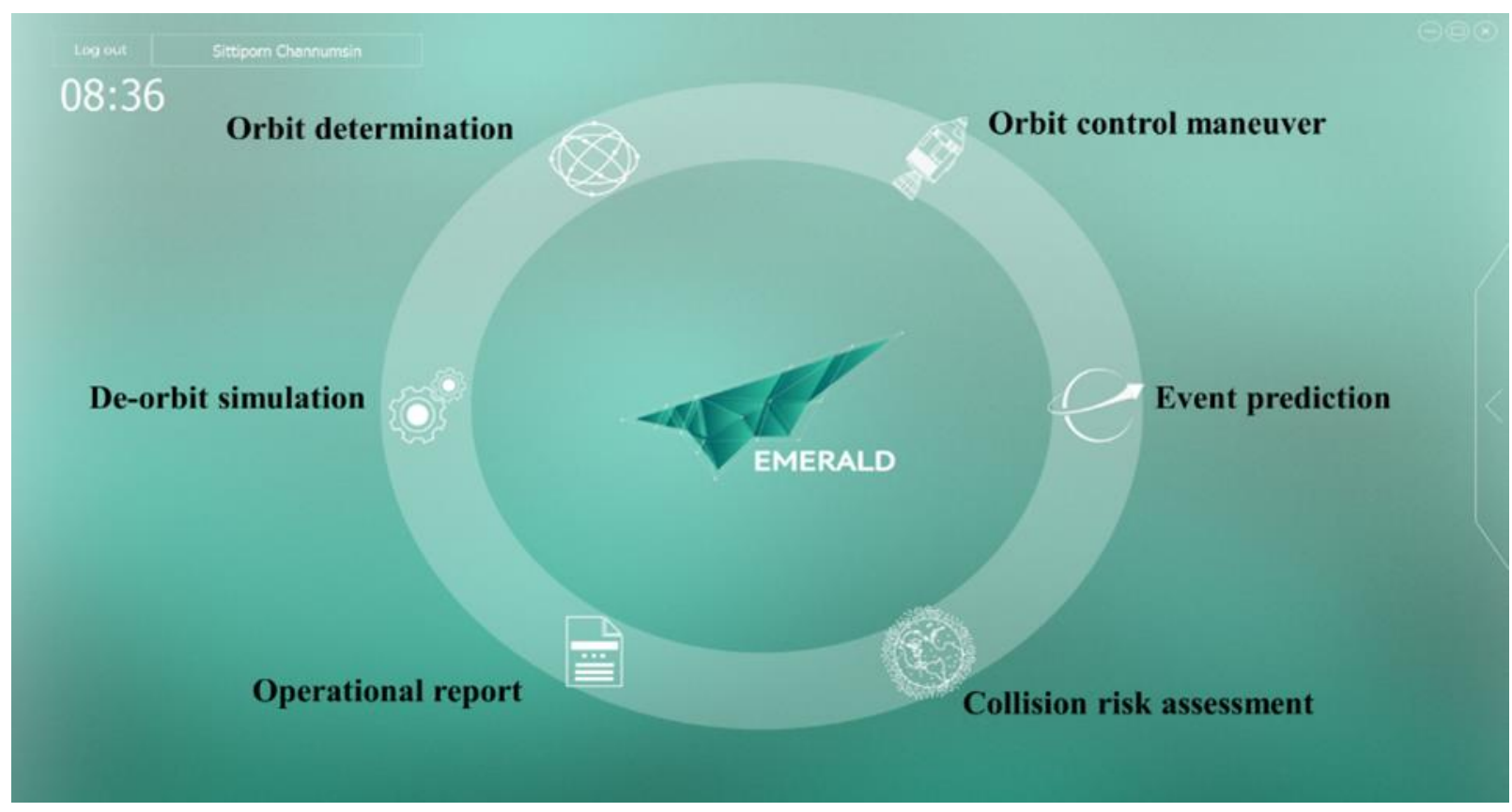

Fig. 1. EMERALD GUI.

\section{Methodology}

In this section, the fundamental of orbital control maneuver can be divided into 2 main parts. The first part is an algorithm to calculate $\Delta V$ for a transfer orbit and station keeping and the second part is to calculate relevant parameters and implements satellite thruster command files. 


\subsection{Transfer Orbit}

The purpose of a transfer orbit is to raise and inject a satellite to the desired orbit after lifting off the launcher's pad. The transfer of satellite might be required to perform several corrections in in-plane or outof-plane to reach the target: semi-major axis (a), inclination (i), eccentricity (e) and to initialize the parameters of the station keeping strategy. The principles of the calculation of in-plane, out-of-plane and combined maneuvers will be described in this section.

\subsubsection{Tangential $\Delta V$ maneuver}

The in-plane maneuvers or "Hohmann transfer" [3] are used to change the semi-major axis of the orbit as shown in Fig. 2. Hohmann transfer can transfer orbit between two circular orbits or two elliptical orbits. The transfer orbit is possibly circular or elliptical depending on the geometry of initial and final orbit. In this study, we focus on both initial and final circular orbits as following Thaichote mission.

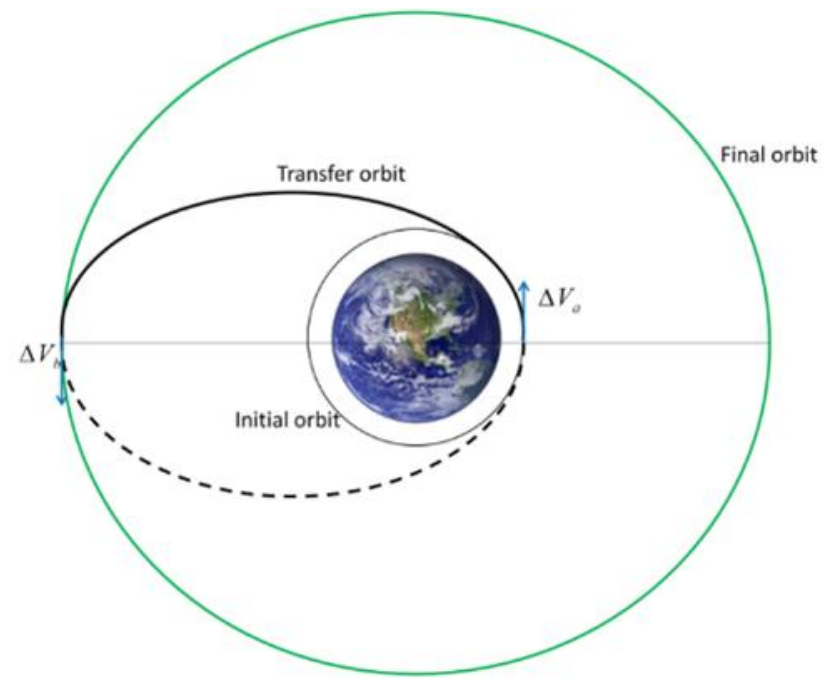

Fig. 2. Hohmann transfer between two circular orbits by using two impulses to minimum change in tangent velocity maneuver.

Before determining the total $\Delta V$ for maneuver, we need to define the $\Delta V$ of each transfer obit $\left(\Delta V_{a}\right.$ and $\Delta V_{b}$ in Fig. 2) by:

$$
\begin{aligned}
& \Delta V_{a}=V_{\text {tran }_{a}}-V_{i} \\
& \Delta V_{b}=V_{f}-V_{\text {tran }_{b}}
\end{aligned}
$$

where

$$
\begin{gathered}
V_{i}=\sqrt{\frac{\mu}{r_{i}}} \\
V_{\text {tran }_{a}}=\sqrt{\frac{2 \mu}{r_{i}}-\frac{\mu}{a_{t}}} \\
V_{f}=\sqrt{\frac{\mu}{r_{f}}}
\end{gathered}
$$




$$
V_{\text {tran }_{b}}=\sqrt{\frac{2 \mu}{r_{f}}-\frac{\mu}{a_{t}}}
$$

Then, $\mu$ is gravitational constant $\left(3.986005 \times 10^{5} \mathrm{~km}^{3} / \mathrm{s}^{2}\right), r_{i}$ is the initial position of a satellite, $r_{f}$ is the final position of a satellite and $a_{t}$ is semi-major axis of a transfer orbit. Finally, we can find the total $\Delta V$ to change the semi-major axis of the orbit as:

$$
\Delta V=\left|\Delta V_{a}\right|-\left|\Delta V_{b}\right|
$$

For a time of flight of a Hohmann transfer, the equation is expressed as:

$$
T_{\text {trans }}=\pi \sqrt{\frac{a_{t}^{3}}{\mu}}
$$

\subsubsection{Out-of-plane $\Delta V$ maneuver}

To change the orbital plane of the satellite (changing inclination only), a satellite requires a component of $\Delta V$ to be perpendicular to the orbital plane as shown in Fig. 3.

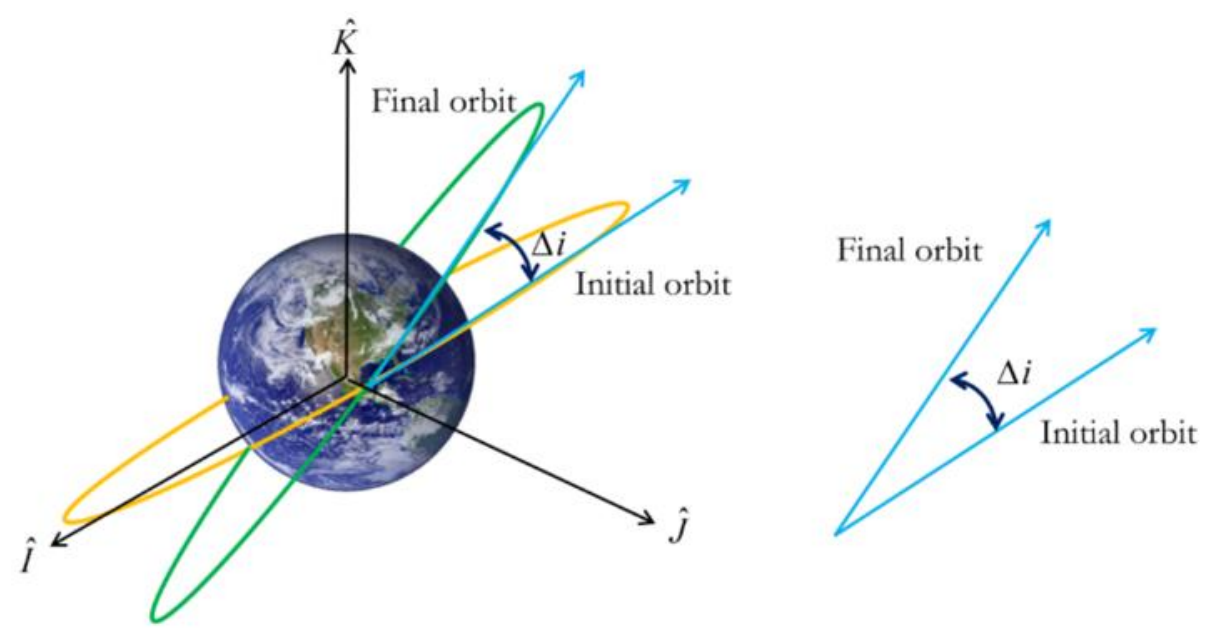

Fig. 3. Orbit transfer for inclination-only change in Earth-centered inertial (ECI) frame $(\hat{I}, \hat{J}, \hat{K})$.

For a circular orbit, $\Delta V$ to change an inclination only is calculated by:

$$
\Delta V=2 V_{i} \sin \left(\frac{\Delta i}{2}\right)
$$

In case of combined maneuvers [4], orbital transfers in Fig. 4 require changes in both the size and the plane of the orbit. The required change in velocity to combine the plane change with the tangential burn is expressed as:

$$
\Delta V=\Delta V_{i}+\Delta V_{f}
$$

where

$$
\Delta V_{i}=\sqrt{V_{i}^{2}+V_{\text {trans }_{a}}^{2}-2 V_{i} V_{\text {trans }_{a}} \cos \left(\Delta i_{i}\right)}
$$




$$
\begin{gathered}
\Delta V_{f}=\sqrt{V_{f}^{2}+V_{\text {trans }_{b}}^{2}-2 V_{f} V_{\text {trans }_{b}} \cos \left(\Delta i_{f}\right)} \\
\Delta i_{i}=s \Delta i \text { and } \Delta i_{f}=(1-s) \Delta i \\
R=\frac{r_{i}}{r_{f}} \text { and } s \approx \frac{1}{\Delta i} \operatorname{Tan}^{-1}\left(\frac{\sin (\Delta i)}{R^{3 / 2}+\cos (\Delta i)}\right)
\end{gathered}
$$

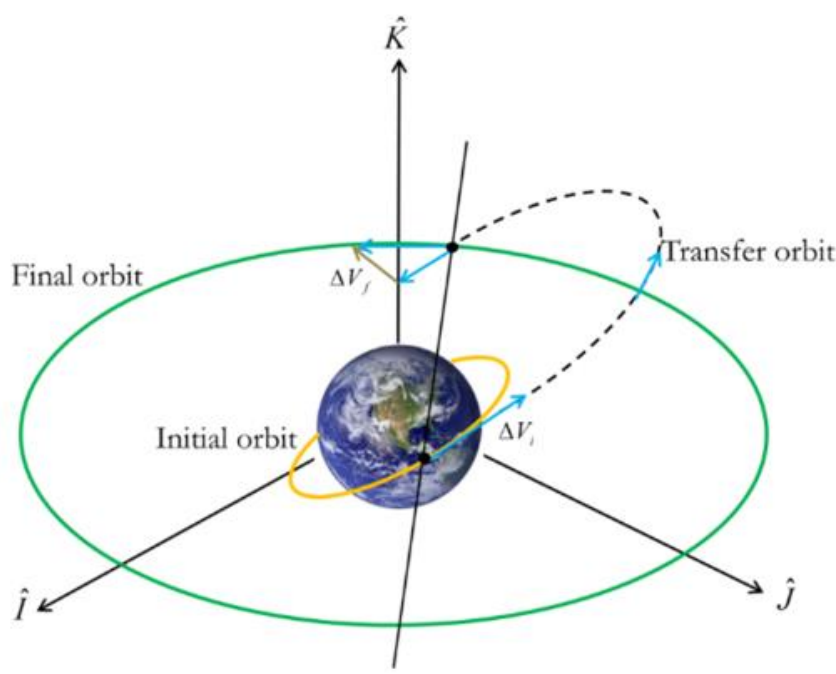

Fig. 4. A transfer orbit from LEO to GEO (Geostationary orbit) in ECI frame by using a combined maneuver.

\subsection{Orbit Control Maneuver for Station Keeping}

The requirement for a classical sun-synchronous station keeping is defined by two parameters: GT and LST. When GT and LST are out of the reference, the orbit control maneuver [4] is required to define the suitable $\Delta V$ to continue the mission.

\subsection{1. $\Delta V$ to control ground track window}

The drift of GT for Thaichote $( \pm 40 \mathrm{~km})$ is from the main effect of semi-major axis change due to an air drag perturbation. Especially, the Sun causes the change of GT to a quadratic function of time. The orbital maneuver will be performed when GT is shifted out of the reference frame in Fig. 5.

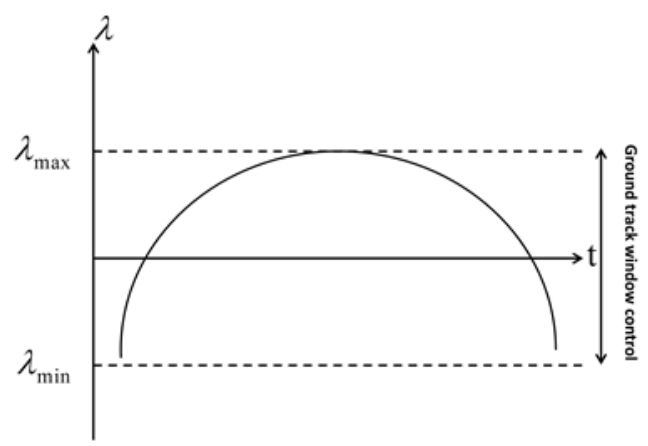

Fig. 5. Ground track control window. 
The orbital velocity adjustment relates to the shift of longitude rate $(\dot{\lambda})$ such that the reduction ratio of an orbital size $(D)$ and the GT drift [4] can be calculated by:

$$
\lambda=\frac{3}{4}\left(\omega_{e}-\dot{\Omega}_{0}\right) \frac{D}{a_{0}} t_{p}^{2}+\dot{\lambda} t_{p}+\lambda_{0}
$$

when $\omega_{e}$ is angular rate of the Earth $\left(7.2921159 \times 10^{-5} \mathrm{rad} / \mathrm{s}\right), \dot{\Omega}_{0}$ is the changing rate of right ascension of ascending node (RAAN), $a_{0}$ is the initial semi-major axis, $t_{p}$ is epoch time, and $\lambda_{0}$ is initial GT. If control period is defined as $T_{c}$, the change of semi-major axis size $(\Delta a)$ can be computed by:

$$
\Delta a=\left(a-a_{0}\right)+\frac{T_{c}}{2} D
$$

where $\Delta V$, which is related to $\Delta a$, can be calculated by using:

$$
\Delta V=\frac{\Delta a \times V}{2 a_{0}(1-e \cos v)}
$$

Given $V \cong \sqrt{\mu / a}$ is orbital velocity, $\mu$ is gravitational constant $\left(3.986005 \times 10^{5} \mathrm{~km}^{3} / \mathrm{s}^{2}\right), a_{0}$ is semi-major axis reference size, $e$ is the eccentricity and $v$ is true anomaly, which is a phase of current position of the orbit. It can be noticed that when a satellite is circular orbit, $(e<<1), \Delta V$ depended on $\Delta a$ only. Therefore, the most efficient desired direction of $\Delta V$ to change $a$ is along-track direction, which is the same as orbital velocity vector, but the $\Delta V$ effects to the eccentricity. To reserve the eccentricity (frozen orbit eccentricity), $\Delta V$ requires to be divided into 2 maneuvers by the $2^{\text {nd }}$ maneuver performed after the $1^{\text {st }}$ maneuver for 180 degrees.

\subsection{2. $\Delta V$ to control local solar time}

The LST evolution depends highly on the inclination evolution. The major disturbance causing to a LST drift is the second term of the earth potential $\left(\mathrm{J}_{2}\right)$ because the fluctuations of this term strongly effect the evolution of the parameters of orbit compared to the next terms of the Earth potential. Thus, the acceleration on the LST effected by the inclination secular drifty [5] is estimated as:

$$
\Delta \ddot{\mathrm{H}}=-\frac{T_{T E}}{T_{S O}}\left[\tan i\left(\frac{d i}{d t}\right)+\frac{7}{2 a} \cdot \frac{d a}{d t}\right]
$$

where $T_{T E}$ is the period of a mean solar day $(86,400 \mathrm{~s}), T_{S O}$ is the sun period equal to $\left(3.15576 \times 10^{7} \mathrm{~s}\right)$, $d a / d t$ is the semi-major axis secular drift and $d i / d t$, the inclination secular drift due to the third body from the Sun, [5] is defined as:

$$
\frac{d i}{d t}=\frac{3 \pi}{2} \cdot \frac{T_{0}}{T_{S O}^{2}} \cdot \sin i \cdot \cos ^{4}\left(\frac{i_{S O}}{2}\right) \cdot \sin \left[4 \pi\left(\frac{H}{T_{T E}}-\frac{1}{2}\right)\right]
$$

where $i_{s o}$ is the inclination of the ecliptic (23.439 degrees). The LST reference of Thaichote is controlled at $10.00 \pm 0.02 \mathrm{a}$.m on descending node (Fig. 6). The orbital inclination $(\Delta i)$ is required to adjust in order to control LST moving to the opposite direction when LST moves to a reference frame. The most efficient direction of $\Delta V[5]$ is the same as angular momentum of the orbit where $\Delta V$ is given by: 


$$
\Delta V=\frac{\Delta i \times V}{\cos (A o L)(1-e \cos v)}
$$

According to Eq. (19) the lowest $\Delta V$ is required when Argument of Latitude (AoL) is 0 or 180 degrees where the satellite crosses the Earth's equator.

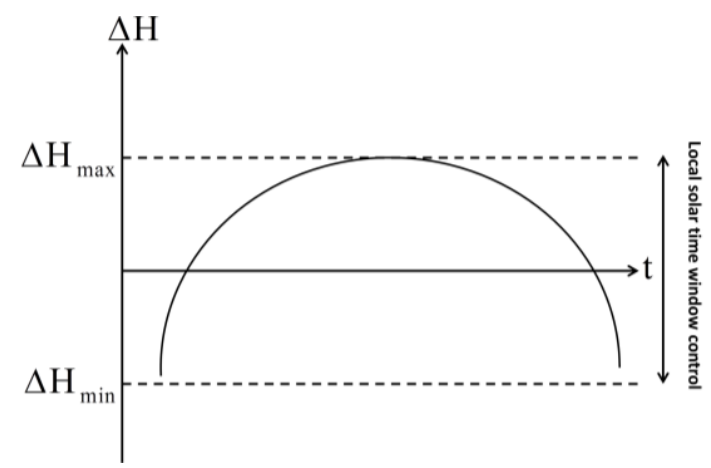

Fig. 6. Local Solar Time control window.

\subsection{Other Maneuver Parameters}

Other maneuver parameters considered to Thaichote maneuver system consist of 5 factors: maneuver duration, maneuver centroid time, thrust level, number of pulses and quaternion.

\subsubsection{Maneuver duration $(\Delta t)$}

During maneuvering, a pressure, volume and propellant mass are changed over time. To simplify the equation, temperature is assumed as constant. Thus, $\Delta V$ [5] will be:

$$
\Delta V=\int_{t_{1}}^{t_{2}} \frac{F(t)}{m(t)} d t
$$

where $F(t)$ is thrust force, $m(t)$ is a satellite mass (platform and propellant). Both parameters are changed over time because of the reduction of volume and propellant pressure during a maneuver. Thus, let $t=\varphi(P)$ and substitute in Eq. (20). The equation can be rewritten in term of a pressure as:

$$
\begin{aligned}
\Delta V & =\int_{\varphi^{-1}\left(t_{1}\right)}^{\varphi^{-1}\left(t_{2}\right)}\left(\frac{F(\varphi(P))}{m(\varphi(P))}(P) \varphi^{\prime}(P)\right) d P \\
& =\int_{P_{1}}^{P_{2}}\left(\frac{F}{m}(P) \varphi^{\prime}(P)\right) d P
\end{aligned}
$$

Then,

$$
\varphi^{\prime}(P)=\frac{d t}{d P}=-\frac{\rho P_{i} V_{i}}{P^{2}} \frac{1}{\left(1-o f f \bmod _{1}\right) q_{1}+\ldots\left(1-o f f \bmod _{1}\right) q_{4}}
$$

where $\rho$ is propellant density, $P_{i}$ and $V_{i}$ are the pressure and volume of propellant before maneuvering, off $\bmod _{i}$ and $q_{i}$ are constants from calibration of gas flow ratio from each engine. Basically, the pressure is decreasing during the maneuver. Therefore, the upper bound of the dichotomy interval is the pressure 
before maneuvering. Finally, after we can determine the final pressure in Eq. (22), the maneuver duration can be computed as:

$$
\Delta t=\int_{P_{1}}^{P_{2}} \varphi^{\prime}(P) d P
$$

\subsubsection{Maneuver centroid time $\left(t_{c}\right)$}

The propulsion is not always constant during maneuver period. It will decrease due to the reduction of the pressure in the tank. The maneuver centroid [5] can be computed by:

$$
t_{c}=\frac{1}{\Delta V} \int_{t_{i}}^{t_{f}} t \frac{F}{m}(t) d t
$$

\subsubsection{Thrust level $(F)$}

The thrust level [5], which is the average of thrust from each engine throughout the pressure change period during maneuver, can be calculated as:

$$
F=\frac{1}{P_{f}-P_{i}} \sum_{i=1}^{N} \int_{P_{i}}^{P_{f}} F_{1}(P) d P
$$

where $P_{i}$ is an initial pressure in tank, $P_{f}$ is a final pressure and $N$ is a number of thrusters.

\subsubsection{Number of pulses (NoP)}

Due to period calculation of flight dynamics simulator software cycle duration is constant at $250 \mathrm{~ms}$, a command transmitter [5] can be done by transmitting pulses as an integer where the overall time consumption can be expressed as:

$$
N o P=\text { Nearest Integer }\left(\begin{array}{l}
{\left[\begin{array}{l}
\text { Theoretical Duration } \\
\text { FSW Cycle Duration }
\end{array}\right]} \\
\times\left[\left(1-\text { off } \bmod \operatorname{coef}_{1}\right)+\ldots\left(1-o f f \bmod \operatorname{coef}_{n}\right)\right]
\end{array}\right)
$$

\subsubsection{Quaternion}

This is how satellite orients to get the direction requirement of $\Delta V$ through Quaternion [6] among the consign frame comparing to Local-Vertical-Local-Horizontal frame (LVLH) and local orbital reference frame ( $\mathrm{T}$ : tangential direction, $\mathrm{N}$ : radial direction, W: normal direction) in Fig. 7 by using:

$$
\begin{aligned}
Q_{-} R 1_{-} t o_{-} R 7= & {\left[Q_{-} R 6_{-} t o_{-} R 7\right] \times\left[Q_{-} R 5_{-} t o_{-} R 6\right] \times\left[Q_{-} R 44_{-} t o_{-} R 5\right] } \\
& \times\left[Q_{-} R 33_{-} t o_{-} R 4\right] \times\left[Q_{-} R 2_{-} t o_{-} R 3\right] \times\left[Q_{-} R 1_{-} t o_{-} R 2\right]
\end{aligned}
$$

where Q_R1_to_R2 is the rotational matrix from LVLH to TNW frame. If the orbit is a complete circle, the 2 coordinates will completely overlay. For elliptical orbit, the angle depends on flight path angle. However, the computation for average angle will be done only one time during orbital maneuver. 
Q_R2_to_R3 is the rotational angle from TNW around $\mathrm{W}$ axis to the direction of $\Delta V$ measuring from $\mathrm{T}$ axis with angle $\alpha$ as shown in Fig. 7(a). If a maneuver is only on the along-track direction, the angle will be 0 and 180 degrees when a maneuver direction is opposite with the orbital velocity.

Q_R3_to_R4 is the rotational angle around $\mathrm{X}$ axis of satellite coordinate at -90 degrees in order to point at $\mathrm{Z}$ direction to centre of earth for orientation mode during standard orbital maneuver.

Q_R4_to_R5 is the rotational angle around $\mathrm{Z}$ axis of satellite coordinate to be $\delta$ angle in order to point at $\mathrm{X}$ axis to the required $\Delta V$ direction. For example, if we require to maneuver in cross-track direction only in order to increase the orbital inclination, which will be 90 degrees.

Q_R5_to_R6 is the rotational angle around $\mathrm{Z}$ axis of satellite coordinate with $\gamma$ angle as shown in Fig. 7(b), corresponding to real orientation of a maneuver.

Q_R6_to_R7 is the rotational angle around $\mathrm{Y}$ axis of satellite coordinate with $\beta$ angle corresponding to a satellite orientation of a maneuver.

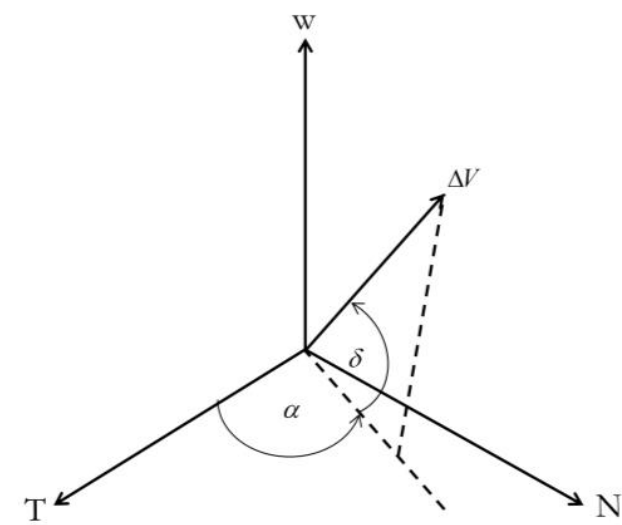

a)

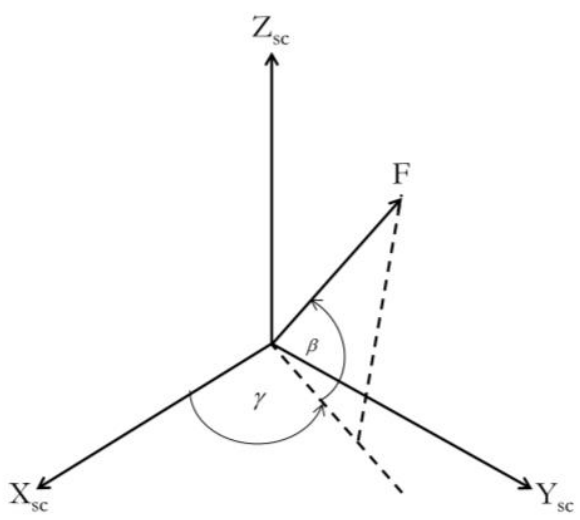

b)

Fig. 7. Satellite's Orientation and Rotation a) TNW frame b) LVLH frame [4].

\subsection{Maneuver Calibration}

Basically, the orbit maneuver do not reach a target completely due to the efficiency of thrust and maneuver control subsystem. The maneuver calibration module purposes to calibrate orbit control maneuver [5] by comparing an actual position from GPS data after maneuvering with a predicted positon by the EMERALD. Thus, we can calculate the maneuver efficiency (ME) and the OCM calibration coefficient for next maneuver as:

$$
\begin{gathered}
M E=\frac{R_{r}-R_{c}}{R_{c}} \\
\operatorname{Coeff}_{u}=\operatorname{Coeff}_{c} \times(1+M E)
\end{gathered}
$$

where $R_{r}$ is an actual position, $R_{c}$ is a predicted position, Coeff $f_{c}$ is the previous calibration coefficient and $\operatorname{Coeff}_{u}$ is the updated calibration coefficient that will be applied in the next maneuver.

\subsection{Propellant Accounting}

The tank volume $\left(V_{\text {tank }}\right)$ of the Thaichote containing both gas (helium, $\left.V_{g}\right)$ and propellant (Hydrazine, $\left.V_{p}\right)$, is constant along the mission. The initial temperature of gas $\left(T_{g, i}\right)$ and the initial pressure of gas $\left(P_{g, i}\right)$ can be received from spacecraft telemetry. The first step to determine the initial gas volume $\left(V_{g, i}\right)$ is determined by: 


$$
V_{g, i}=V_{\mathrm{tank}}-\frac{M_{p, i}}{\rho_{p}}
$$

where $M_{g, i}$ is the initial propellant mass before maneuvering, $\rho_{p}$ is propellant density and tank volume $\left(V_{\text {tank }}\right)$ of Thaichote is $0.1 \mathrm{~m}^{3}$. Based on the gas law equation, the initial gas pressure [7] is determined by:

$$
P_{g, i} V_{g, i}=n_{g} R T_{g, i}
$$

where $n_{g}$ is moles and $R$ is universal gas constant (0.0821 L.atm/mol.K) [7]. We can find the final gas volume $\left(V_{g, f}\right)$ with the universal gas law [7]:

$$
V_{g, f}=\frac{P_{g, i} V_{g, i}}{T_{g, i}} \frac{T_{g, f}}{P_{g, f}}
$$

Finally, the final propellant volume and mass $\left(V_{p, f}, M_{p, f}\right)$ are given by:

$$
\begin{gathered}
V_{p, f}=V_{\text {tank }}-V_{g, f} \\
M_{p, f}=V_{p, f} \rho_{p}
\end{gathered}
$$

\section{Results and Discussions}

Fig. 8 shows the OCM module that has 5 sub-modules: orbit control maneuver, maneuver plan generation, maneuver calibration, transfer orbit and propellant accounting. The results of EMERALD are compared by using the previous events analysed by Quartz++.

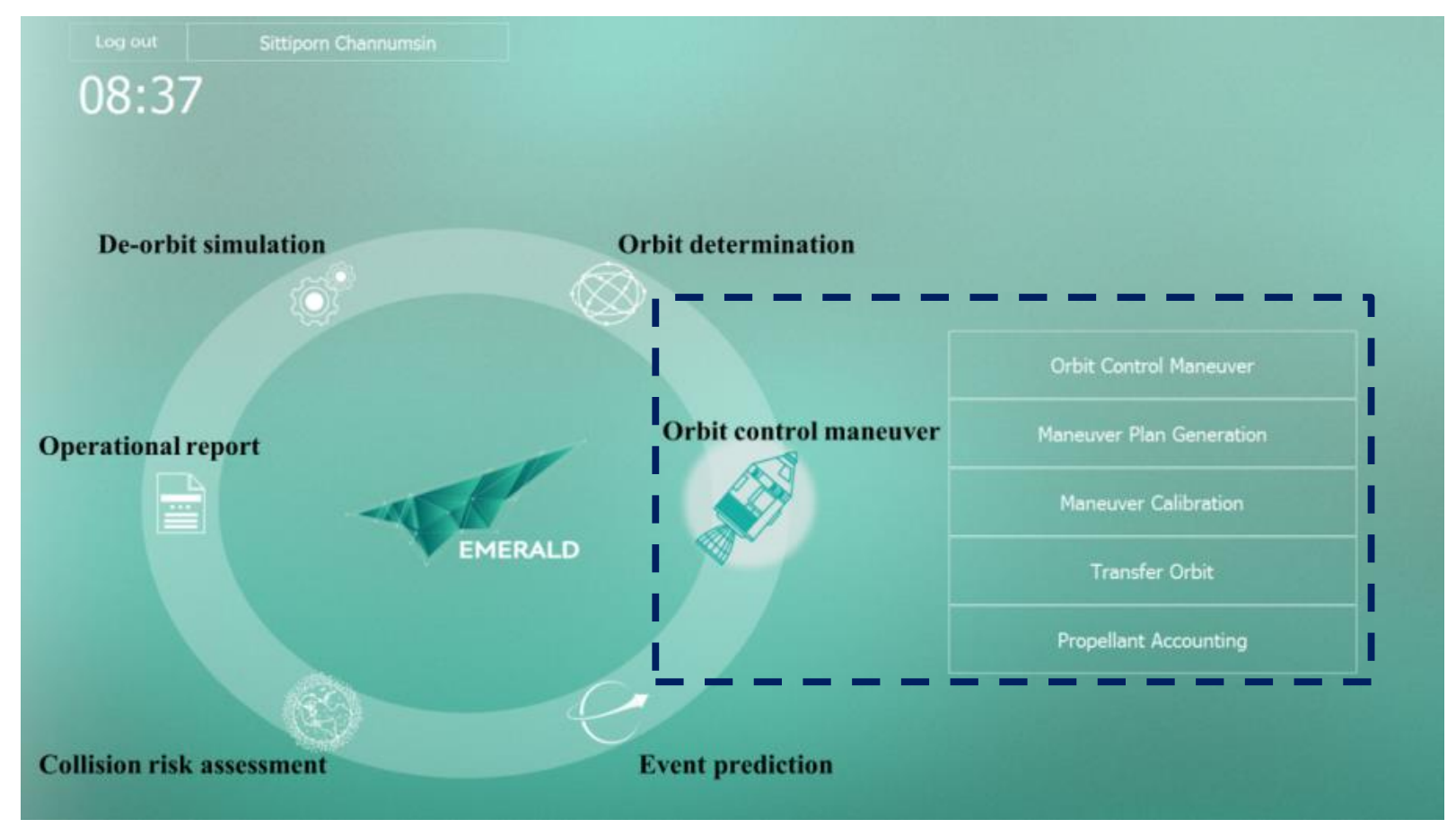

Fig. 8. Orbit control maneuver module. 


\subsection{Orbit Control Maneuver (OCM)}

Fig. 9 presents OCM module implementing to compute the orbital maneuver vectors to change semi-major axis or inclination or both including the results of orbital elements, GT and LST after maneuver. Table 1 shows the different comparison of 4 events (two events for semi-major axis correction and two others for inclination correction). The maximum error of semi-major axis and inclination are $2.94 \%$ and $2.81 \%$ on 13 Mar 2014 and 23 Nov 2010. Both GT error and LST error of all maneuvers are under threshold (GT error: $\pm 40 \mathrm{~km}$ and LST error: $10.00 \pm 0.02$ a.m). Fig. 10 shows the example of the results of semi-major axis, ground track error and local solar time after maneuvering to correct semi-major axis.

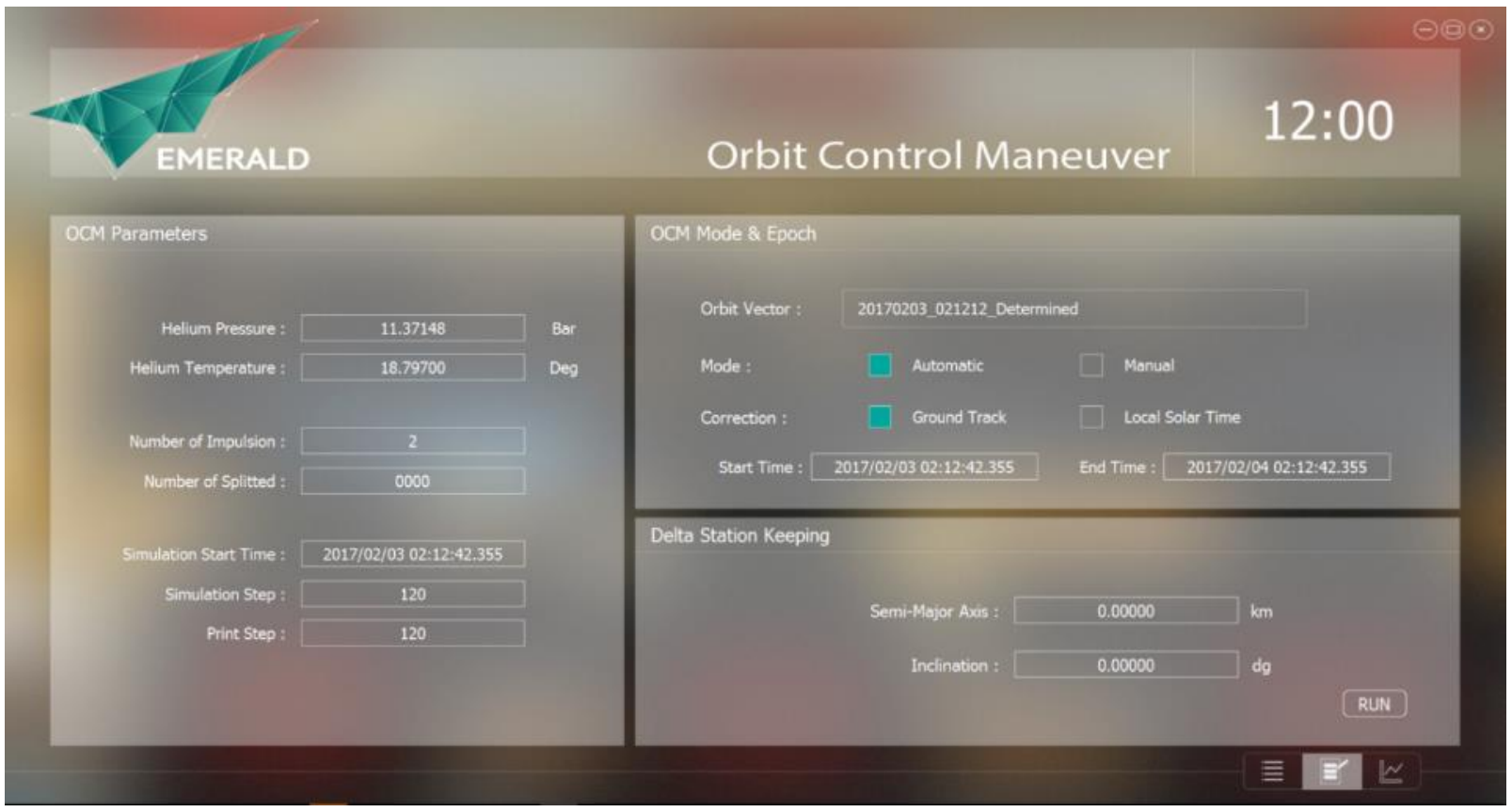

Fig. 9. Orbit control maneuver module.

Table 1. Percentage error of EMERALD by comparing with Quartz++.

\begin{tabular}{ccc|ccc}
\hline $\begin{array}{c}\text { Difference } \\
\text { Percent (\%) }\end{array}$ & \multicolumn{2}{c|}{ Semi-major axis correction } & $\begin{array}{c}\text { Difference } \\
\text { Percent (\%) }\end{array}$ & \multicolumn{2}{c}{ Inclination correction } \\
\hline Date & 31 Jan 2013 & 13 Mar 2014 & Date & 23 Nov 2010 & 16 Jan 2013 \\
a (\%) & 1.168 & 2.940 & $i(\%)$ & 2.810 & 2.040 \\
GT error(km) & 15.75 to 17.28 & 8.62 to 9.73 & GT error(km) & 20.12 to 21.53 & 14.82 to 16.13 \\
LST(a.m.) & $10.01-10.02$ & $9.59-10.01$ & LST(a.m.) & $10.00-10.01$ & $9.58-9.59$ \\
$\Delta V(\%)$ & 0.107 & 0.729 & $\Delta V(\%)$ & 0.024 & 0.207 \\
$\Delta t(\%)$ & 2.379 & 3.010 & $\Delta t(\%)$ & 1.105 & 0.687 \\
$F(\%)$ & 0.043 & 0.053 & $F(\%)$ & 0.090 & 0.023 \\
NoP(\%) & 2.310 & 3.180 & NoP(\%) & 1.109 & 0.691 \\
\hline
\end{tabular}




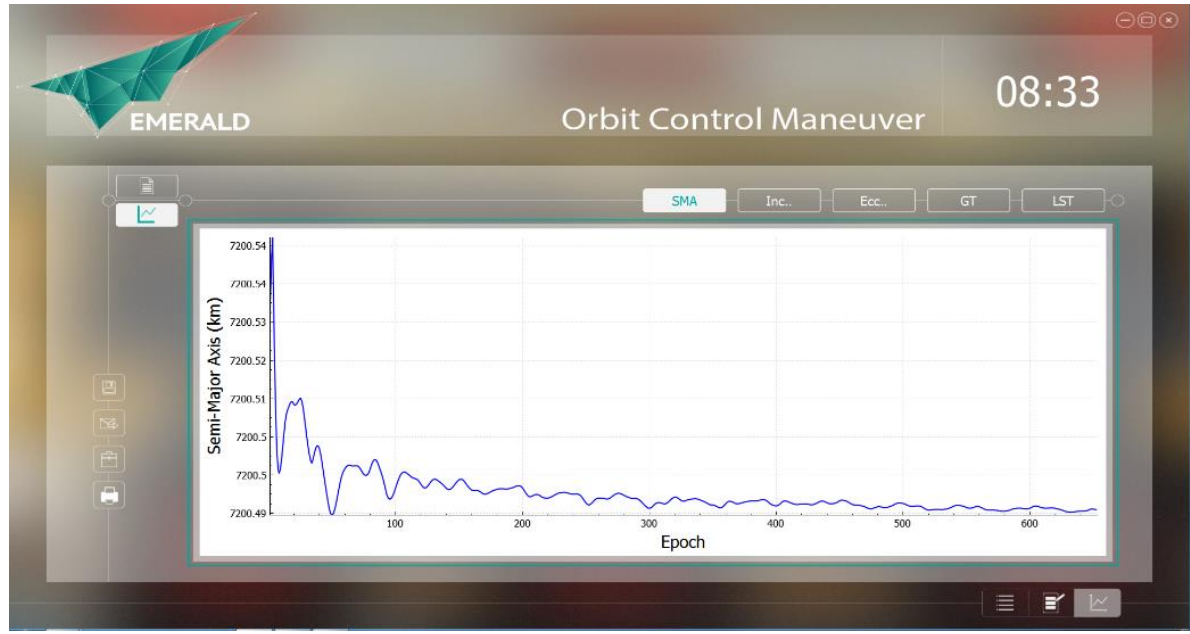

a)

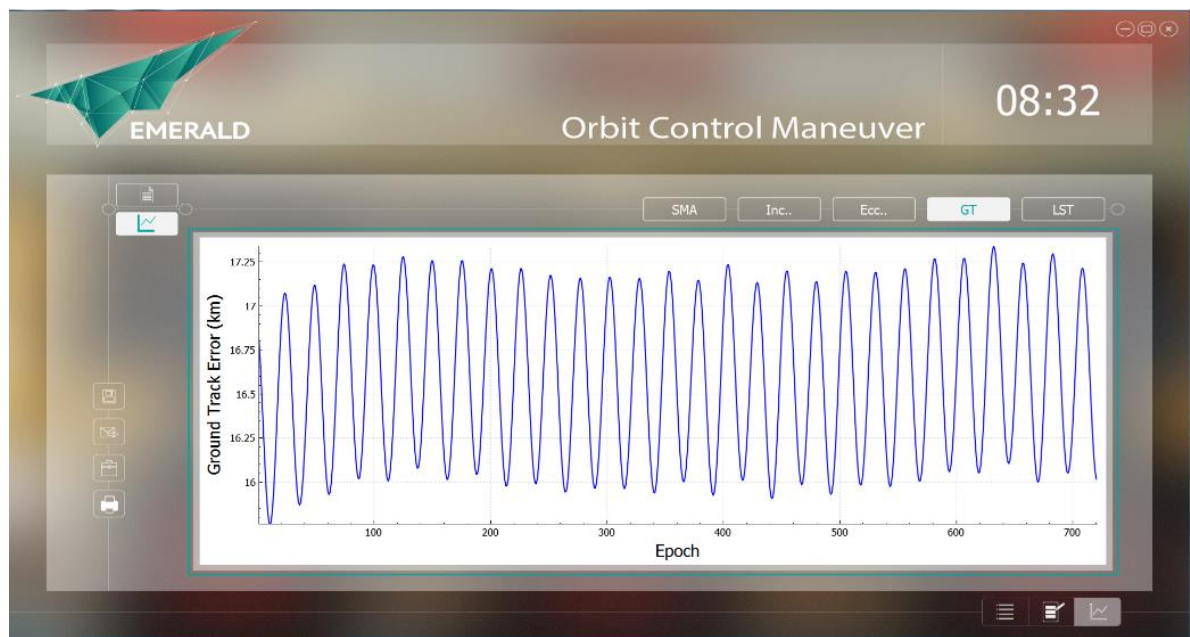

b)

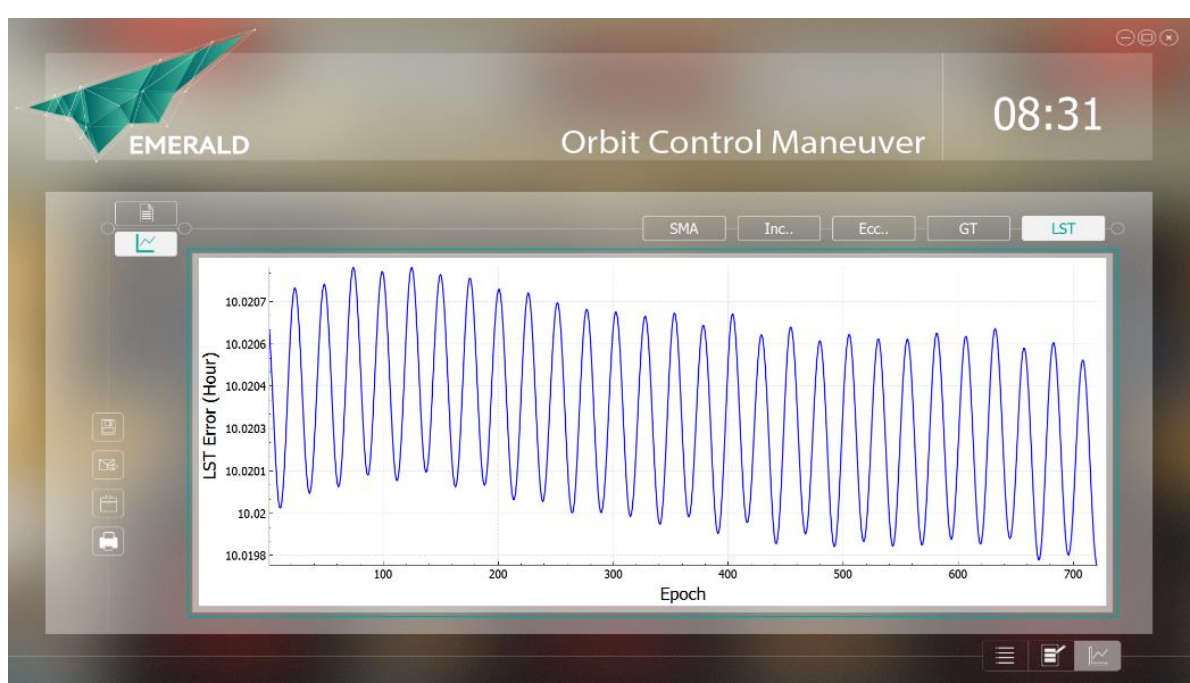

c)

Fig. 10. Example of results after maneuvering to correct semi-major axis a) Semi-major axis evolution b) Ground track error c) Local solar time error.

3.2. Maneuver Plan Generation and Maneuver Calibration 
Fig. 11 shows the GUI of the maneuver plan generation module to generate a file for satellite maneuver system command and the maneuver calibration to monitor the OCM efficiency and compute a calibration coefficient for next maneuver. In this investigation, we test the data on 31 Jan 2013 and 13 Mar 2014. Table 2 shows the small difference in time to command IOC B board (thrust control board) and reaction wheels when comparing with Quartz++. In Table 3, the OCM efficiency and calibration coefficient of EMERALD are very close to the results of Quartz++.

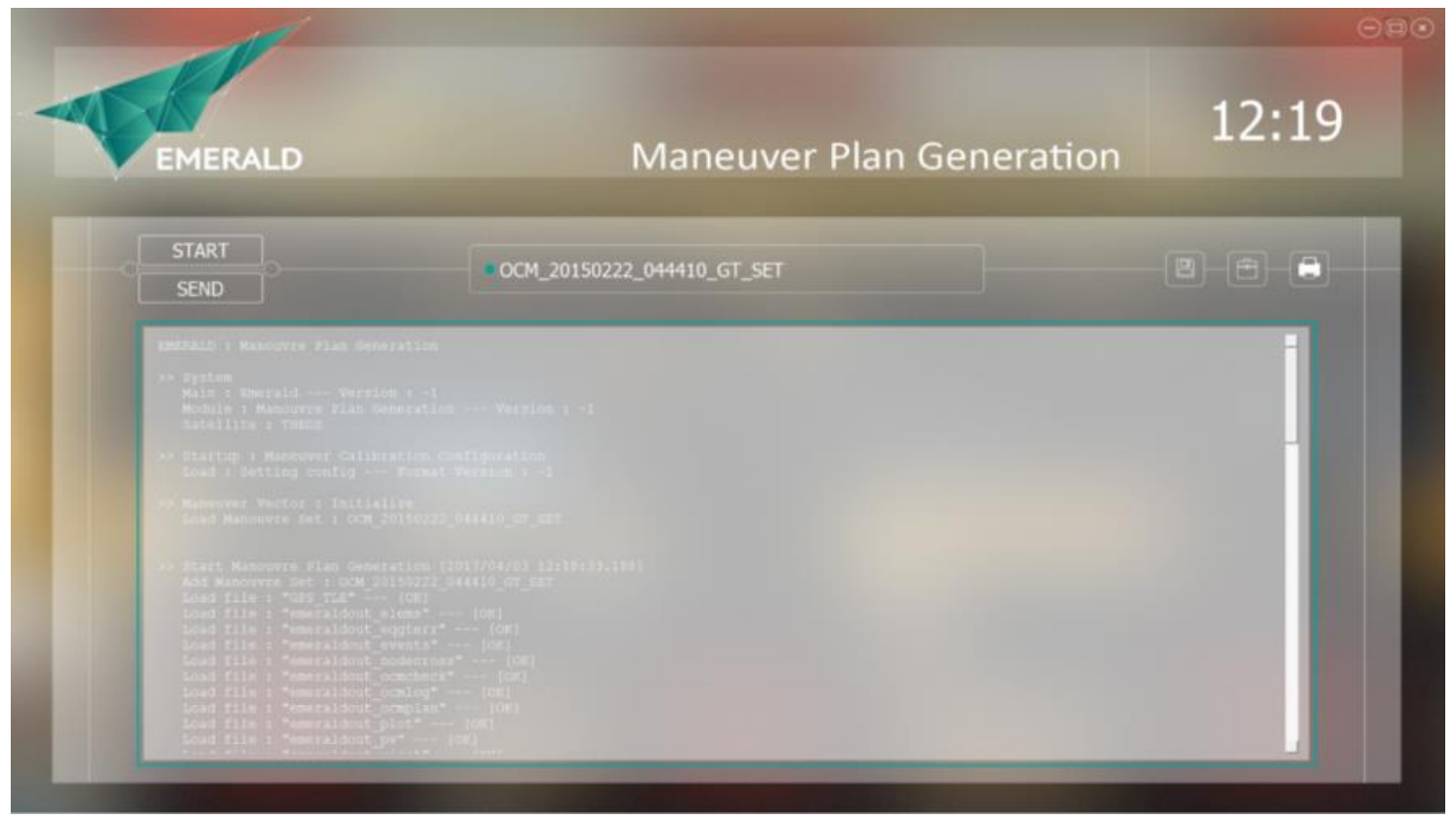

a)

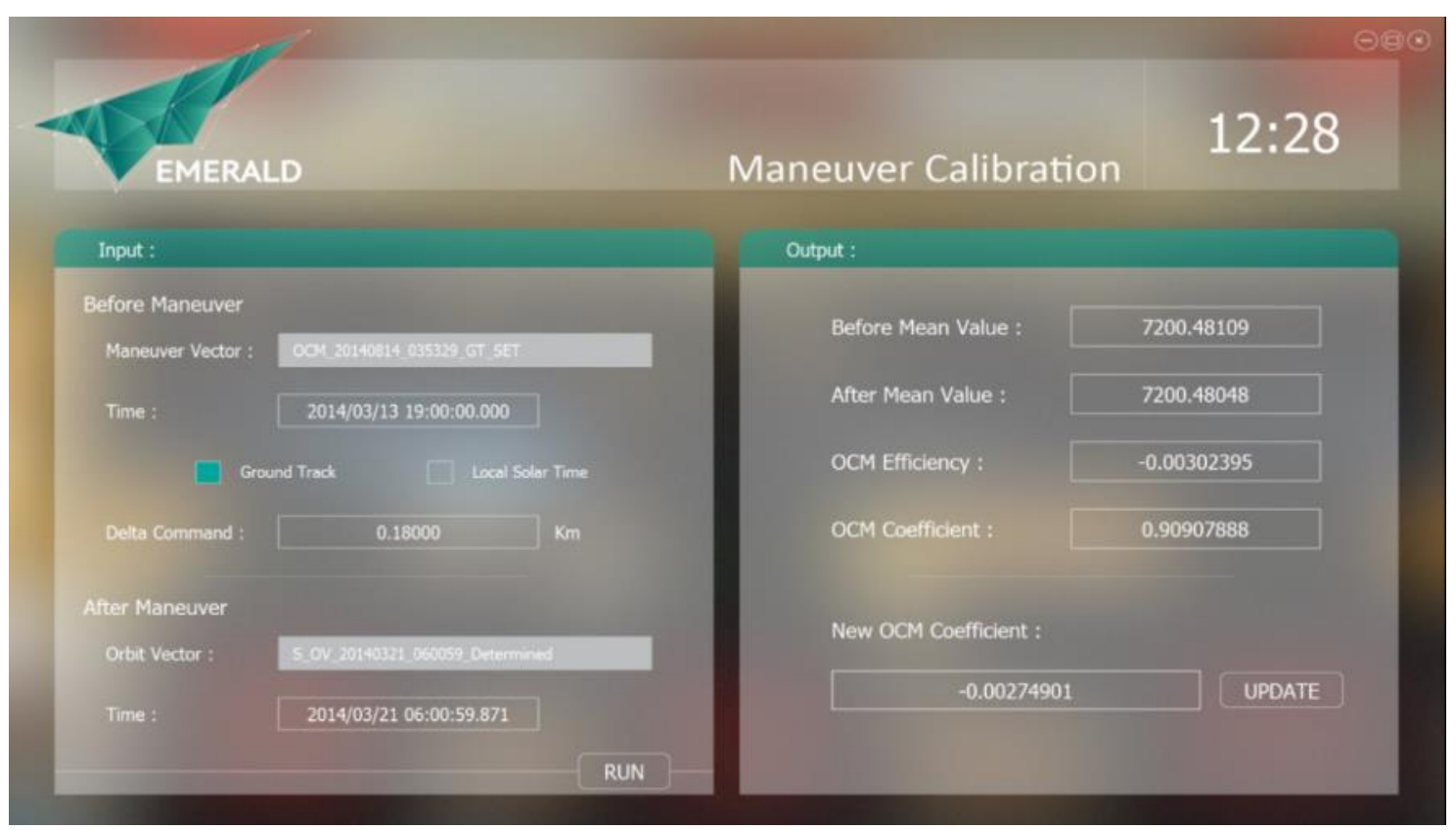

b)

Fig. 11. Maneuver plan generation GUI a) maneuver plan generation module b) maneuver calibration module. 
Table 2. The difference of the operating period to command on/off of IOC B board (thruster control board) and on/off reaction wheels.

\begin{tabular}{|c|c|c|c|c|}
\hline $\begin{array}{c}\text { Date } \\
\Delta a \text { target }(\mathrm{km})\end{array}$ & \multicolumn{2}{|c|}{$\begin{array}{c}\text { 31 Jan } 2013 \\
0.483\end{array}$} & \multicolumn{2}{|c|}{$\begin{array}{c}13 \text { Mar } 2014 \\
0.163\end{array}$} \\
\hline Maneuver order & $1^{\text {st }}$ & $2^{\text {nd }}$ & $1^{\text {st }}$ & $2^{\text {nd }}$ \\
\hline Switch on IOC B board(s) & 23 & 87 & 4 & 97 \\
\hline Switch off IOC B board(s) & 19 & 97 & 14 & 91 \\
\hline Thruster Configuration(s) & 23 & 87 & 4 & 97 \\
\hline Modify wheel allocation (s) & \multicolumn{2}{|c|}{23} & \multicolumn{2}{|c|}{4} \\
\hline Return wheel allocation (s) & \multicolumn{2}{|c|}{94} & \multicolumn{2}{|c|}{91} \\
\hline
\end{tabular}

Table 3. The percent of OCM efficiency and OCM calibration coefficient for next maneuver.

\begin{tabular}{ccccc}
\hline Date & \multicolumn{2}{c}{ 31 Jan 2013 } & \multicolumn{2}{c}{ 13 Mar 2014 } \\
\hline & Quartz++ & EMERALD & Quartz++ & EMERALD \\
\hline OCM efficiency (\%) & 99.754 & 99.729 & 99.874 & 99.849 \\
OCM calibration coefficient & 0.9153 & 0.8926 & 0.9068 & 0.8845 \\
\hline
\end{tabular}

\subsection{Transfer Orbit}

Transfer orbit module in Fig. 12 is to compute an orbital change and construct a data set after maneuvering. This module offers 3 modes for an orbital adjustment, which are repeat ground track (RGT), frozen orbit (FRO), and combination of both (CMB). This module has the capability to continue maneuvers up to 28 times/simulation. For this test, we investigate the lift off from a launcher pad of Thaichote to the desired orbit mission. The results in Table 4 show that the tiny drift of orbital elements between EMERALD and Quartz++. These differences do not effect the Thaichote operation. 


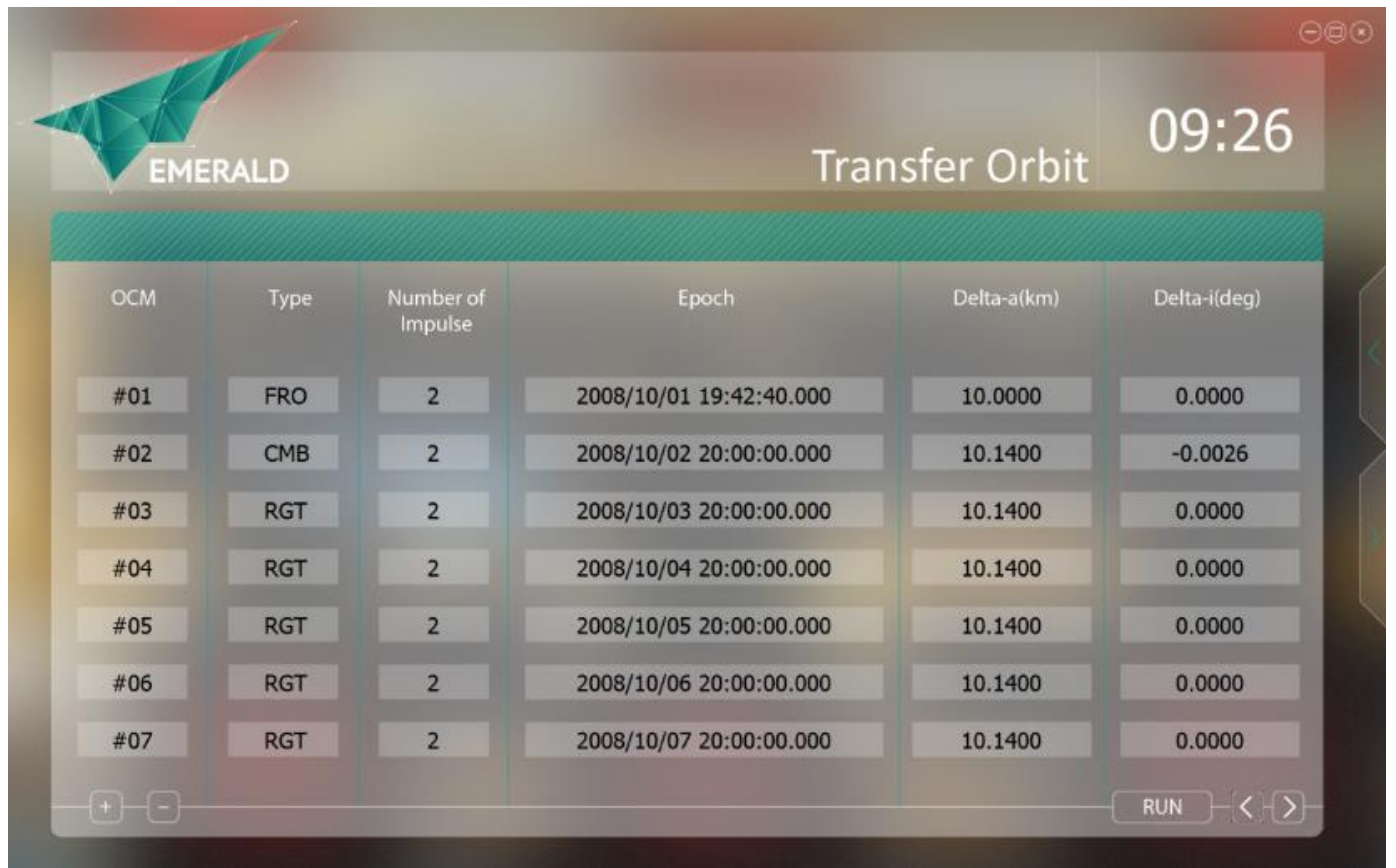

a)

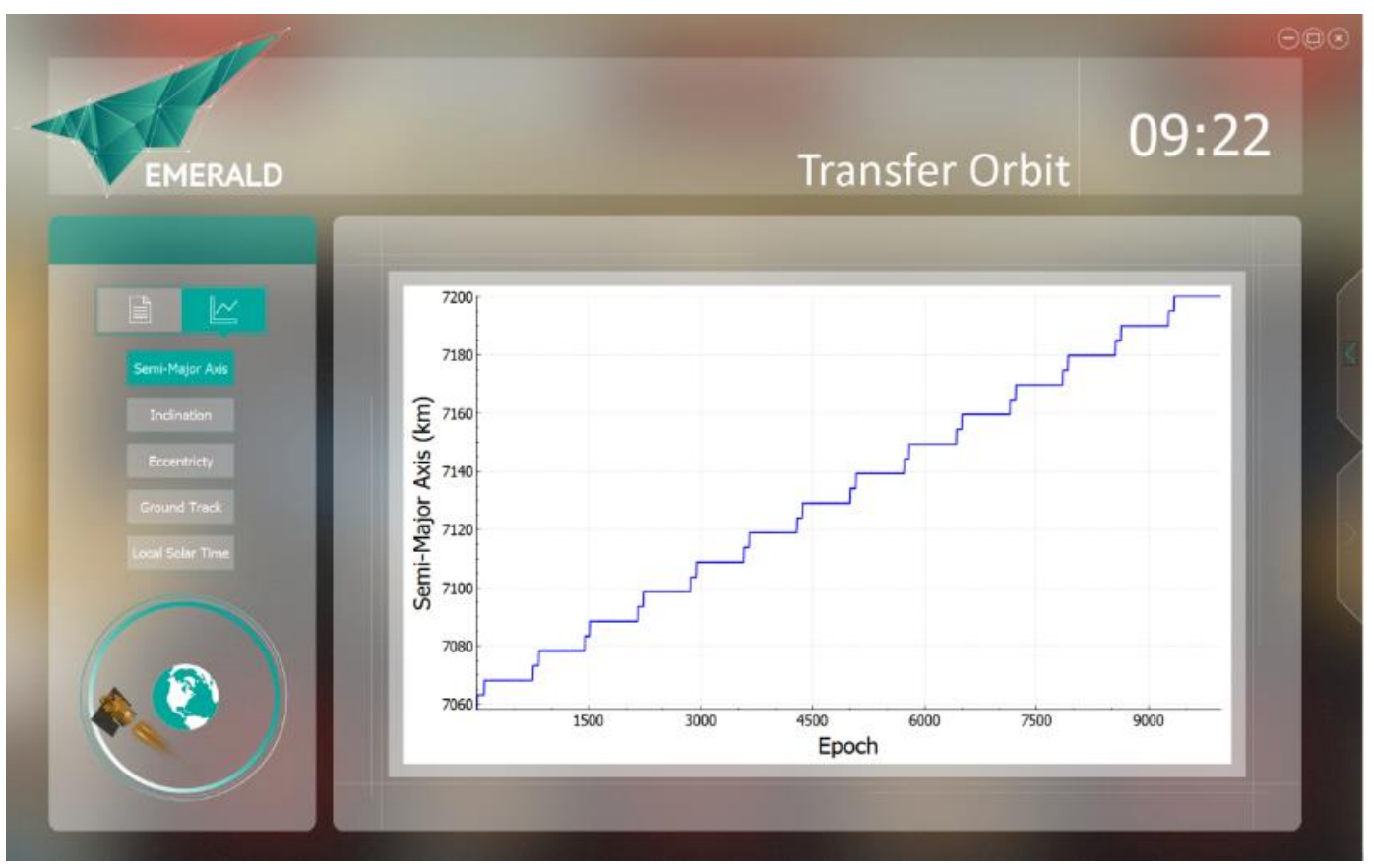

b)

Fig. 12. Transfer orbit GUI a) transfer orbit input b) semi-major axis evolution after 14 maneuvers.

Table 4. Comparison of the simulation results of orbital elements, GT and LST between EMERALD and Quartz++ after maneuvering.

\begin{tabular}{cccc}
\hline Results & EMERALD & Quartz++ & Difference \\
\hline $\mathrm{a}(\mathrm{km})$ & $7,200.614$ & $7,200.629$ & -0.015 \\
$i(\mathrm{deg})$ & 98.781 & 98.779 & 0.002 \\
E & 0.00135 & 0.00114 & 0.00021 \\
GT $(\mathrm{km})$ & 4.37 & 4.13 & 0.24 \\
LST & $09: 59: 24$ & $09: 59: 16$ & $8(\mathrm{~s})$ \\
\hline
\end{tabular}




\subsection{Propellant Accounting}

Propellant accounting module in Fig. 13 is a module to compute the propellant volume in a tank. The computation requires two inputs from a satellite's telemetry file (temperature and pressure inside the tank). The test results are provided in Table 5. The difference of the propellant masses between EMERALD and Quartz++ before and after orbit maneuver is very small and the absolute maximum difference $(|\Delta|)$ of the propellant consumption is $2.398 \mathrm{~g}$ of the maneuver on $16 \mathrm{Jan} 2014$.

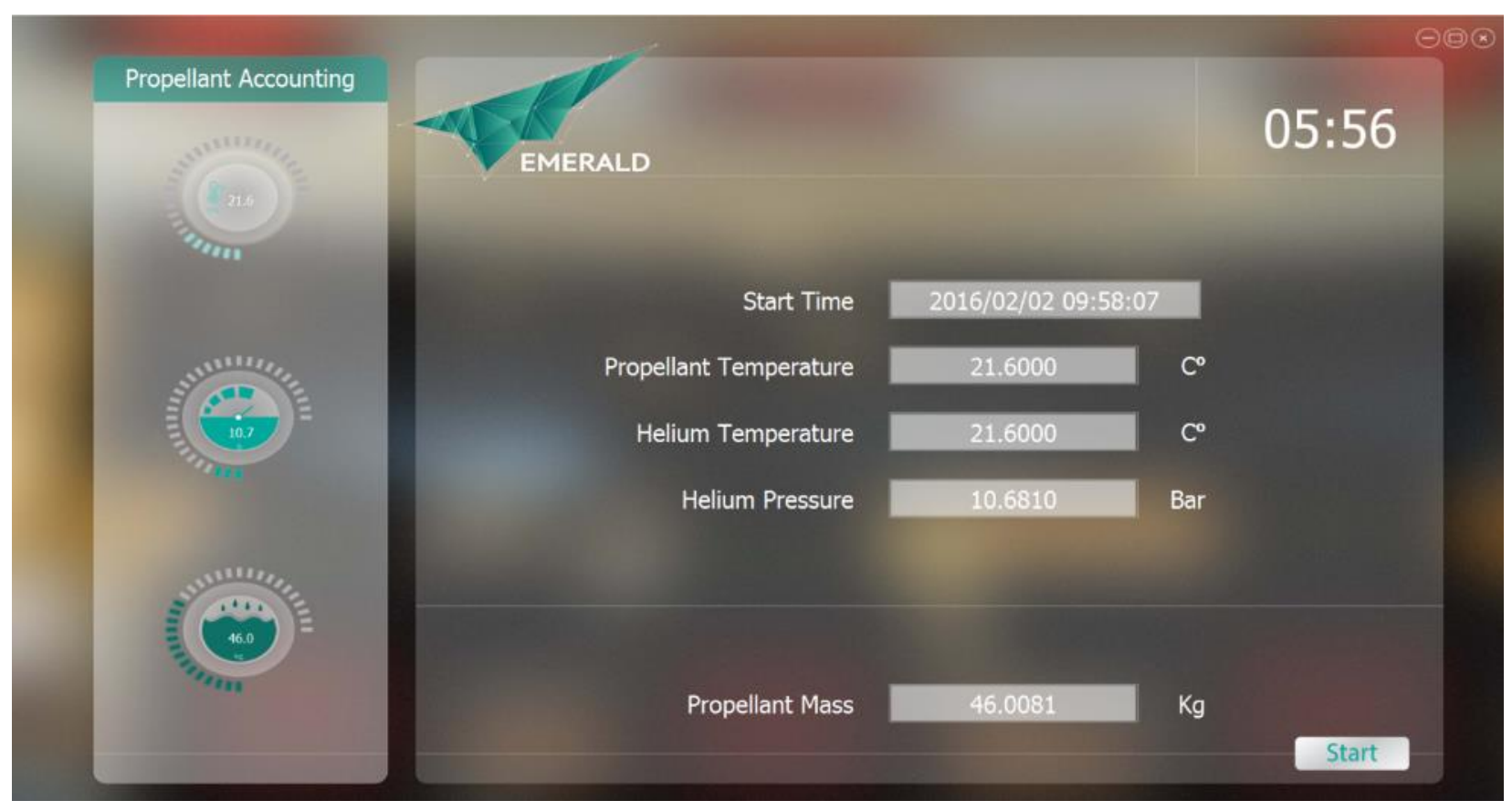

Fig. 13. Propellant accounting module.

Table 5. The difference $(\Delta)$ of propellant accounting calculation between EMERALD and Quartz++.

\begin{tabular}{cccc}
\hline Date & $\begin{array}{c}\Delta \text { Propellant before } \\
\text { maneuver(g) }\end{array}$ & $\begin{array}{c}\Delta \text { Propellant after } \\
\text { maneuver(g) }\end{array}$ & $|\Delta|$ Propellant consumption(g) \\
\hline 10 Feb 2010 & 29.105 & 29.132 & 0.027 \\
23 Nov 2010 & 32.760 & 31.055 & 1.704 \\
19 Apr 2011 & 31.092 & 31.155 & 0.062 \\
3 Apr 2013 & 31.093 & 31.236 & 0.014 \\
16 Jan 2014 & 32.570 & 34.068 & 2.398 \\
\hline
\end{tabular}

\section{Conclusion}

The orbital trajectory analysis tool, EMERALD, has been developed to support Thaichote mission. This paper presents the validation results of OCM modules. This module can design a transfer orbit, control the ground track and local solar time, calculate propellant remaining and generate maneuver plan to command a satellite. The validation results of OCM modules show that this tool provides the sufficient high accuracy and the capacity to construct a data set of the satellite orbital maneuver including convenient GUI that is very useful for the operation.

Future development of the tool aims to improve the efficiency of the maneuver calibration by considering the uncertainties due to perturbations (e.g. solar radiation pressure and air drag) and thruster conditions (e.g. unbalance or magnitude constraints). This tool will be implemented with the full operation in term of the flight dynamics software to be substituted for Quartz++. Next, the development of a realtime tracking providing current position of a satellite, collision avoidance warning and other essential 
parameters of the routine operation will be developed and integrated with EMERALD. The tools will be implemented in the future mission analysis for new GISTDA satellites.

\section{Acknowledgements}

This work was funded by Geo-Informatics and Space Technology Development Agency (Public Organization): GISTDA and the authors thank Siriphat Pomyen for their valuable and helpful comments, which have improved this paper.

\section{References}

[1] P. Navakitkanok, A. H. Gicquel, A. Pimnoo, and S. Jaturut, "THEOS orbit maintenance: assessment of 2 years operations," in The 22nd International symposium on Space Flight Dynamics, 2011, Brazil.

[2] P. Udomthanatheera, P. Navakitkanok, and M. Aorpimai, "The development of satellite orbit analysis and determination processing system: EMERALD," in 38th Electrical Engineering Conference, 2015, Ayutthya, Thailand.

[3] W. Hohmann, "The attainability of heavenly bodies (translation of Die Erreichbarkeit der Himmelskorper)," in NASA Tecbnical Translation F-44. National Aeronautics and Space Administration: Washington, DC, Nov. 1960.

[4] D. A. Vallado, Fundamentals of Astrodynamics and Applications. New York: Microcosm Press, 2007.

[5] F. Raballand, Quartz++ LEO FDS User Guide. EADS ASTRIUM, 2007.

[6] H. Schaub and J. L. Junkins, Analytical Mechanics of Space Systems. American Institute of Aeronautics and Astronautics, 2003.

[7] D. W. Rogers, Concise Physical Chemistry. New Jersey: John Wiley \& Sons, 2011. 